\title{
INVISCID MODELS GENERALIZING THE 2D EULER AND THE SURFACE QUASI-GEOSTROPHIC EQUATIONS
}

\author{
DONGHO CHAE ${ }^{1}$, PETER CONSTANTIN ${ }^{2}$ AND JIAHONG WU ${ }^{3}$
}

\begin{abstract}
Any classical solution of the 2D incompressible Euler equation is global in time. However, it remains an outstanding open problem whether classical solutions of the surface quasi-geostrophic (SQG) equation preserve their regularity for all time. This paper studies solutions of a family of active scalar equations in which each component $u_{j}$ of the velocity field $u$ is determined by the scalar $\theta$ through $u_{j}=\mathcal{R} \Lambda^{-1} P(\Lambda) \theta$ where $\mathcal{R}$ is a Riesz transform and $\Lambda=(-\Delta)^{1 / 2}$. The $2 \mathrm{D}$ Euler vorticity equation corresponds to the special case $P(\Lambda)=I$ while the SQG equation to the case $P(\Lambda)=\Lambda$. We develop tools to bound $\|\nabla u\|_{L^{\infty}}$ for a general class of operators $P$ and establish the global regularity for the $\log \log$-Euler equation for which $P(\Lambda)=(\log (I+\log (I-\Delta)))^{\gamma}$ with $0 \leq \gamma \leq 1$. In addition, a regularity criterion for the model corresponding to $P(\Lambda)=\Lambda^{\beta}$ with $0 \leq \beta \leq 1$ is also obtained.
\end{abstract}

\section{Introduction AND STATEMENTS OF the MAIN RESUlts}

This paper studies solutions of the active scalar equation

$$
\left\{\begin{array}{l}
\partial_{t} \theta+u \cdot \nabla \theta=0, \quad x \in \mathbb{R}^{d}, t>0, \\
u=\left(u_{j}\right), \quad u_{j}=\mathcal{R}_{l} \Lambda^{-1} P(\Lambda) \theta, \quad 1 \leq j, l \leq d,
\end{array}\right.
$$

where $\theta=\theta(x, t)$ is a scalar function of $x \in \mathbb{R}^{d}$ and $t \geq 0, u$ denotes a velocity field with its component $u_{j}(1 \leq j \leq d)$ given by a Riesz transform $\mathcal{R}_{l}$ applied to $\Lambda^{-1} P(\Lambda) \theta$. Here the operators $\Lambda=(-\Delta)^{\frac{1}{2}}, P(\Lambda)$ and $\mathcal{R}_{l}$ are defined through their Fourier transforms, namely

$$
\widehat{\Lambda f}(\xi)=|\xi| \widehat{f}(\xi), \quad \widehat{P(\Lambda) f}(\xi)=P(|\xi|) \widehat{f}(\xi), \quad \widehat{\mathcal{R}_{l} f}(\xi)=\frac{i \xi_{l}}{|\xi|} \widehat{f}(\xi)
$$

where $1 \leq l \leq d$ is an integer, $\widehat{f}$ or $\mathcal{F}(f)$ denotes the Fourier transform,

$$
\widehat{f}(\xi)=\mathcal{F}(f)(\xi)=\frac{1}{(2 \pi)^{d / 2}} \int_{\mathbb{R}^{d}} e^{-i x \cdot \xi} f(x) d x .
$$

Our consideration is restricted to $P$ satisfying the following Assumption.

Assumption 1.1. The symbol $P=P(|\xi|)$ assumes the following properties:

(1) $P$ is continuous on $\mathbb{R}^{d}$ and $P \in C^{\infty}\left(\mathbb{R}^{d} \backslash\{0\}\right)$;

(2) $P$ is radially symmetric;

(3) $P=P(|\xi|)$ is nondecreasing in $|\xi|$;

(4) There exist two constants $C$ and $C_{0}$ such that

$$
\sup _{2^{-1} \leq|\eta| \leq 2}\left|\left(I-\Delta_{\eta}\right)^{n} P\left(2^{j}|\eta|\right)\right| \leq C P\left(C_{0} 2^{j}\right)
$$

2000 Mathematics Subject Classification. 35Q53, 35B35, 35B65, 76D03.

Key words and phrases. generalized surface quasi-geostrophic equation, global regularity. 


$$
\text { for any integer } j \text { and } n=1,2, \cdots, 1+\left[\frac{d}{2}\right] \text {. }
$$

We remark that (4) in Assumption 1.1 is a very natural condition on symbols of Fourier multiplier operators and is similar to the main condition in the Mihlin-Hörmander Multiplier Theorem (see e.g. [83, p.96]). For notational convenience, we also assume that $P \geq 0$. Some special examples of $P$ are

$$
\begin{aligned}
& P(\xi)=\left(\log \left(1+|\xi|^{2}\right)\right)^{\gamma} \quad \text { with } \gamma \geq 0, \\
& P(\xi)=\left(\log \left(1+\log \left(1+|\xi|^{2}\right)\right)\right)^{\gamma} \quad \text { with } \gamma \geq 0, \\
& P(\xi)=|\xi|^{\beta} \quad \text { with } \beta \geq 0, \\
& P(\xi)=\left(\log \left(1+|\xi|^{2}\right)\right)^{\gamma}|\xi|^{\beta} \quad \text { with } \gamma \geq 0 \text { and } \beta \geq 0 .
\end{aligned}
$$

A particularly important case of (1.1) is the 2D active scalar equation

$$
\left\{\begin{array}{l}
\partial_{t} \theta+u \cdot \nabla \theta=0, \quad x \in \mathbb{R}^{2}, t>0, \\
u=\nabla^{\perp} \psi \equiv\left(-\partial_{x_{2}} \psi, \partial_{x_{1}} \psi\right), \quad-\Lambda^{2} \psi=P(\Lambda) \theta
\end{array}\right.
$$

which generalizes the 2D Euler vorticity equation

$$
\left\{\begin{array}{l}
\partial_{t} \omega+u \cdot \nabla \omega=0, \\
u=\nabla^{\perp} \psi, \quad \Delta \psi=\omega
\end{array}\right.
$$

and the surface quasi-geostrohic (SQG) equation

$$
\left\{\begin{array}{l}
\partial_{t} \theta+u \cdot \nabla \theta=0 \\
u=\nabla^{\perp} \psi, \quad-\Lambda \psi=\theta
\end{array}\right.
$$

The 2D Euler equation has been extensively studied and its global regularity has long been established (see e.g. [16], 60] and [66]). The SQG equation and its dissipative counterpart have recently attracted a lot of attention and numerous efforts have been devoted to the global regularity and related issues concerning their solutions (see e.g. [1], [2], [3], [5], 6], [7], 8], [9], [10], [1], [12], [13], [14], [15], [17], [18], [19], [20], [21], [22], [23], [24], [26], [27], [28], [29], [30], [31], [32], [33], [34], [35], [36], [37], [38], [39], [40], [41], [42], [43], [44], [45], [46], [47], [48], [49], [50], [51], [52], [53], [54], [55], [56], [57], [58], [59], 60], 61], 62], 63], 64], 65], 67], 66], 69], [70], [71], 72], [73], [74, [75], 76], [77], [78], [79], [80], [81], [82], [85], [86], [87], [88], [89], [90], [91], [91], [92], [93], [94], [95], [96], [97], [98], [99], [100], [101]).

The goal of this paper is to understand the global regularity issue concerning solutions of (1.1) with a given initial datum

$$
\theta(x, 0)=\theta_{0}(x), \quad x \in \mathbb{R}^{d} .
$$

The key quantity involved in this issue is $\|\nabla u\|_{L^{\infty}}$. Tools are developed here to bound $\left\|\Delta_{j} \nabla u\right\|_{L^{p}}$ and $\left\|S_{N} \nabla u\right\|_{L^{p}}$ when a vector field $u: \mathbb{R}^{d} \rightarrow \mathbb{R}^{d}$ is related to a scalar function $\theta$ by

$$
(\nabla u)_{j k}=\mathcal{R}_{l} \mathcal{R}_{m} P(\Lambda) \theta
$$

where $1 \leq j, k, l, m \leq d,(\nabla u)_{j k}$ denotes the $(j, k)$-th entry of $\nabla u$ and $\mathcal{R}_{l}$ and $\mathcal{R}_{m}$ denote the Riesz transforms. Here $\Delta_{j}$ with $j \geq-1$ denotes the Fourier localization operator 
and

$$
S_{N}=\sum_{j=-1}^{N-1} \Delta_{j} .
$$

The precise definitions of $\Delta_{j}$ and $S_{N}$ are provided in Appendix $\mathrm{A}$. The assumption that $u$ is divergence-free is not used in deriving these bounds. The bounds obtained here are summarized in the following theorem.

Theorem 1.2. Let $u: \mathbb{R}^{d} \rightarrow \mathbb{R}^{d}$ be a vector field. Assume that $u$ is related to a scalar $\theta$ by

$$
(\nabla u)_{j k}=\mathcal{R}_{l} \mathcal{R}_{m} P(\Lambda) \theta,
$$

where $1 \leq j, k, l, m \leq d,(\nabla u)_{j k}$ denotes the $(j, k)$-th entry of $\nabla u, \mathcal{R}_{l}$ denotes the Riesz transform, and $P$ obeys Assumption 1.1. Then, for any integers $j \geq 0$ and $N \geq 0$,

$$
\begin{aligned}
\left\|S_{N} \nabla u\right\|_{L^{p}} & \leq C_{p, d} P\left(C_{0} 2^{N}\right)\left\|S_{N} \theta\right\|_{L^{p}}, \quad 1<p<\infty \\
\left\|\Delta_{j} \nabla u\right\|_{L^{q}} & \leq C_{d} P\left(C_{0} 2^{j}\right)\left\|\Delta_{j} \theta\right\|_{L^{q}}, \quad 1 \leq q \leq \infty \\
\left\|S_{N} \nabla u\right\|_{L^{\infty}} & \leq C_{d}\|\theta\|_{L^{1} \cap L^{\infty}}+C_{d} N P\left(C_{0} 2^{N}\right)\left\|S_{N+1} \theta\right\|_{L^{\infty}},
\end{aligned}
$$

where $C_{p, d}$ is a constant depending on $p$ and $d$ only and $C_{d} s$ ' depend on $d$ only.

We remark that in general the constant $C_{p, d}$ grows linearly with respect to $p$ and thus (1.6) does not follow for $p=\infty$. With these bounds at our disposal, we are able to establish global regularity results covering two special cases of $P$. The first result is for (1.1) with $P(|\xi|)=\left(\log \left(1+\log \left(1+|\xi|^{2}\right)\right)\right)^{\gamma}$. For the simplicity of our presentation here, we state the result for the $2 \mathrm{D}$ case of (1.1), namely

$$
\left\{\begin{array}{l}
\partial_{t} \theta+u \cdot \nabla \theta=0, \\
u=\nabla^{\perp} \psi, \quad \Delta \psi=(\log (1+\log (1-\Delta)))^{\gamma} \theta,
\end{array}\right.
$$

which we call the Loglog-Euler equation. Although any classical solution $\theta$ of (1.9) obeys the global a priori bound

$$
\|\theta(\cdot, t)\|_{L^{p}} \leq\|\theta(\cdot, 0)\|_{L^{p}} \quad \text { for any } \quad 1 \leq p \leq \infty,
$$

the regularity of the velocity $u$ recovered from the relation

$$
u=\nabla^{\perp} \psi, \quad \Delta \psi=(\log (1+\log (1-\Delta)))^{\gamma} \theta
$$

is worse than in the case of the $2 \mathrm{D}$ Euler equation. Nevertheless we are able to obtain the global regularity for (1.9) with $0 \leq \gamma \leq 1$.

Theorem 1.3. Consider the initial-value problem (1.9) and (1.5) with $\gamma$ and $\theta_{0}$ satisfying

$$
0 \leq \gamma \leq 1, \quad \theta_{0} \in L^{1}\left(\mathbb{R}^{2}\right) \cap L^{\infty}\left(\mathbb{R}^{2}\right) \cap B_{q, \infty}^{s}\left(\mathbb{R}^{2}\right)
$$

where $2<q \leq \infty$ and $s>1$. Then the initial-value problem (1.9) and (1.5) has a unique global solution $\theta$ satisfying,

$$
\theta \in L^{\infty}\left([0, \infty) ; B_{q, \infty}^{s}\left(\mathbb{R}^{2}\right)\right), \quad \nabla u \in L^{\infty}\left([0, \infty) ; B_{q, \infty}^{1+s_{1}}\left(\mathbb{R}^{2}\right)\right),
$$

where $s_{1}<s$. 
The general version of Theorem 1.3, namely the global regularity result for (1.1) will be stated in Section 3. Here $B_{q, \infty}^{s}$ denotes an inhomogeneous Besov space. The definition of a general Besov space $B_{p, q}^{s}$ is provided in Appendix A. Even though $\theta_{0} \in B_{q, \infty}^{s}$ implies $\theta_{0} \in L^{\infty}$, the condition on $\theta_{0}$ is written as in (1.10) to emphasize the importance of $L^{\infty}$ assumption. The global regularity stated in the Besov space setting in Theorem 1.3 can be converted into a global regularity statement in Sobolev spaces. Combining Theorem 1.3 and the embedding relations

$$
W_{q}^{r} \hookrightarrow B_{q, \infty}^{r} \hookrightarrow B_{q, \min \{2, q\}}^{r_{1}} \hookrightarrow W_{q}^{r_{1}}, \quad r>r_{1},
$$

we can conclude that any initial data in $W_{q}^{r}$ with $2<q \leq \infty$ and $r>1$ would yield a global solution in $W_{q}^{r_{1}}$ for any $r_{1}<r$.

Theorem 1.3 is proven by combining the Besov space techniques and the following extrapolation inequality.

Proposition 1.4. Let $u: \mathbb{R}^{d} \rightarrow \mathbb{R}^{d}$ be a vector field. Assume that $u$ is related to a scalar $\theta$ by

$$
(\nabla u)_{j k}=\mathcal{R}_{l} \mathcal{R}_{m}(\log (I+\log (I-\Delta)))^{\gamma} \theta
$$

where $\gamma \geq 0,1 \leq j, k, l, m \leq d,(\nabla u)_{j k}$ denotes the $(j, k)$-th entry of $\nabla u$ and $\mathcal{R}_{l}$ and $\mathcal{R}_{m}$ denote the Riesz transforms. Then, for any $1 \leq q \leq \infty$ and $s>d / q$,

$$
\|\nabla u\|_{L^{\infty}} \leq\|\theta\|_{L^{1} \cap L^{\infty}}+C\|\theta\|_{L^{\infty}} \log \left(1+\|\theta\|_{B_{q, \infty}^{s}}\right)\left(\log \left(1+\log \left(1+\|\theta\|_{B_{q, \infty}^{s}}\right)\right)\right)^{\gamma}
$$

where $C$ is a constant that depends on $d, q$ and $s$ only.

The second special case studied here is when $P(|\xi|)=|\xi|^{\beta}$ with $0 \leq \beta \leq 1$. Our aim is to understand how the parameter $\beta$ affects the regularity of solutions to the initial-value problem

$$
\left\{\begin{array}{l}
\partial_{t} \theta+u \cdot \nabla \theta=0 \\
u=\nabla^{\perp} \psi, \quad-\Lambda^{2} \psi=\Lambda^{\beta} \theta
\end{array}\right.
$$

where $0 \leq \beta \leq 1$. The evolution of patch-like initial data under (1.12) has previously been studied in [33]. Clearly (1.12) bridges the 2D Euler and the SQG equation. It is hoped that this study would shed light on the global regularity issue concerning the SQG equation.

It is unknown if all classical solutions of (1.12) conserve their regularity for all time except in the case of the 2D Euler equation. In order to deal with the global regularity for (1.12), it suffices to obtain a suitable bound for $\|\nabla u\|_{L^{\infty}\left(\mathbb{R}^{2}\right)}$. Intuitively, the relation

$$
u=-\nabla^{\perp} \Lambda^{-2+\beta} \theta
$$

implies that $\|\nabla u\|_{L^{\infty}\left(\mathbb{R}^{2}\right)}$ can be bounded more or less by a bound for $\Lambda^{\beta} \theta$. In fact, this intuitive idea can be made rigorous and is reflected in the following logarithmic Hölder inequality

$$
\|S\|_{L^{\infty}} \leq C\|\theta\|_{C^{\beta}} \ln \left(1+\|\theta\|_{C^{\sigma}}\right)+C\|\theta\|_{L^{q}}, \quad \sigma>\beta, q>1,
$$

where $S$ denotes the symmetric part of $\nabla u$ and $C^{\beta}$ the Hölder space. This inequality, together with a bound for the back-to-labels map determined by $u$, allows us to obtain the following regularity criterion. 
Theorem 1.5. Consider (1.12) with $0 \leq \beta \leq 1$. Let $\theta$ be a solution of (1.12) corresponding to the data $\theta_{0} \in C^{\sigma}\left(\mathbb{R}^{2}\right) \cap L^{q}\left(\mathbb{R}^{2}\right)$ with $\sigma>1$ and $q>1$. Let $T>0$. If $\theta$ satisfies

$$
\int_{0}^{T}\|\theta(\cdot, t)\|_{C^{\beta}\left(\mathbb{R}^{2}\right)} d t<\infty
$$

then $\theta$ remains in $C^{\sigma}\left(\mathbb{R}^{2}\right) \cap L^{q}\left(\mathbb{R}^{2}\right)$ on the time interval $[0, T]$.

This criterion especially establishes the global regularity for the 2D Euler equation and reduces to the well-known criterion for the SQG equation when $\beta=1$ (see [23]).

The rest of this paper is organized as follows. Section 2 is devoted to the bounds in Theorem 1.2 and Proposition 1.4. Theorem 1.3 and its general version, the global regularity result for (1.1) are stated and proven in Section 3, Section 4 details the proof of Theorem 1.5. Appendix $\mathrm{A}$ provides the definition of Besov spaces and some related facts.

\section{Bounds FOR $\left\|\Delta_{j} \nabla u\right\|_{L^{q}},\left\|S_{N} \nabla u\right\|_{L^{q}}$ AND $\|\nabla u\|_{L^{\infty}}$}

This section derives the bounds stated in Theorem 1.2 and proves the logarithmic interpolation inequality presented in Proposition 1.4.

We make use of Mihlin and Hörmander Multiplier Theorem (see [83, p.96]) in the proof of (1.6). This theorem is recalled first.

Theorem 2.1. Suppose that $Q(\xi)$ is of class $C^{k}$ in the complement of the origin of $\mathbb{R}^{d}$, where $k>\frac{d}{2}$ is an integer. Assume also that

$$
\left|D^{\alpha} Q(\xi)\right| \leq B|\xi|^{-|\alpha|}, \quad \text { whenever }|\alpha| \leq k .
$$

Then $Q \in \mathcal{M}_{q}, 1<q<\infty$. That is, $\left\|T_{Q} f\right\|_{L^{q}} \leq C_{q}\|f\|_{L^{q}}$, where $T_{Q}$ is defined by

$$
\widehat{T_{Q} f}(\xi)=Q(\xi) \widehat{f}(\xi) \text {. }
$$

For further reference, we rewrite (1.6) as a proposition.

Proposition 2.2. Let $u: \mathbb{R}^{d} \rightarrow \mathbb{R}^{d}$ be a vector field. Assume that $u$ is related to a scalar $\theta$ by

$$
(\nabla u)_{j k}=\mathcal{R}_{l} \mathcal{R}_{m} P(\Lambda) \theta
$$

where $1 \leq j, k, l, m \leq d,(\nabla u)_{j k}$ denotes the $(j, k)$-th entry of $\nabla u, \mathcal{R}_{l}$ denotes the Riesz transform and $P$ obeys Assumption 1.1. Then, for any integer $N \geq 0$,

$$
\left\|S_{N} \nabla u\right\|_{L^{p}} \leq C_{p, d} P\left(C_{0} 2^{N}\right)\left\|S_{N} \theta\right\|_{L^{p}}, \quad 1<p<\infty
$$

where $C_{p, d}$ is a constant depending on $p$ and $d$ only.

Proof. As detailed in Appendix $\underline{\mathrm{A}}$, the symbol of $S_{N}$ is $\psi\left(\xi / 2^{N}\right)$ with $\psi$ satisfying

$$
\psi \in C_{0}^{\infty}\left(\mathbb{R}^{d}\right), \quad \operatorname{supp} \psi \subset B\left(0, \frac{11}{12}\right), \quad \psi(\xi)=1 \text { for }|\xi| \leq \frac{3}{4} .
$$

It follows from (2.2) that

$$
\left(\widehat{S_{N} \nabla u}\right)_{j k}(\xi)=Q(\xi) P\left(C_{0} 2^{N}\right) \widehat{S_{N} \theta}(\xi)
$$


where $Q(\xi)$ is supported on $|\xi| \leq(11 / 12) 2^{N}$ and, for $|\xi| \leq(11 / 12) 2^{N}$,

$$
Q(\xi)=-\frac{\xi_{l} \xi_{m}}{|\xi|^{2}} \frac{P(|\xi|)}{P\left(C_{0} 2^{N}\right)} .
$$

To apply Theorem 2.1 , we verify (2.1). Clearly, for any $\alpha$ with $|\alpha|=0,1, \cdots, 1+\left[\frac{d}{2}\right]$,

$$
\left|D^{\alpha} \frac{\xi_{l} \xi_{m}}{|\xi|^{2}}\right| \leq C|\xi|^{-|\alpha|} .
$$

In addition, for any $\xi \neq 0$, there is an integer $j$ such that $\xi=2^{j} \eta$ with $2^{-1} \leq|\eta| \leq 2$. Trivially, for $\xi$ in the support of $Q, j \leq N$. It is easy to see that Condition (4) in Assumption 1.1 implies that

$$
\sup _{2^{-1} \leq|\eta| \leq 2}\left|\left(-\Delta_{\eta}\right)^{n} P\left(2^{j}|\eta|\right)\right| \leq C P\left(C_{0} 2^{j}\right)
$$

for $n=0,1, \cdots, 1+\left[\frac{d}{2}\right]$. Then,

$$
\begin{aligned}
\left|\left(-\Delta_{\xi}\right)^{n} \frac{P(|\xi|)}{P\left(C_{0} 2^{N}\right)}\right| & =\left|\left(-\Delta_{\eta}\right)^{n} \frac{2^{-2 n j} P\left(2^{j}|\eta|\right)}{P\left(C_{0} 2^{N}\right)}\right| \\
& \leq|\eta|^{2 n}\left|2^{j} \eta\right|^{-2 n} \frac{P\left(C_{0} 2^{j}\right)}{P\left(C_{0} 2^{N}\right)} \\
& \leq|\eta|^{2 n}|\xi|^{-2 n} .
\end{aligned}
$$

This verifies (2.1) (2.3) then follows as a consequence of Theorem 2.1.

For the sake of clarity, we restate (1.7) in Theorem 1.2 as a proposition.

Proposition 2.3. Let $u: \mathbb{R}^{d} \rightarrow \mathbb{R}^{d}$ be a vector field. Assume that $u$ is related to a scalar $\theta$ by

$$
(\nabla u)_{j k}=\mathcal{R}_{l} \mathcal{R}_{m} P(\Lambda) \theta
$$

where $1 \leq j, k, l, m \leq d,(\nabla u)_{j k}$ denotes the $(j, k)$-th entry of $\nabla u$ and $\mathcal{R}_{l}$ denotes the Riesz transform. Here $P$ obeys Assumption 1.1. Then, for any integer $N \geq 0$,

$$
\left\|\Delta_{N} \nabla u\right\|_{L^{q}} \leq C_{d} P\left(C_{0} 2^{N}\right)\left\|\Delta_{N} \theta\right\|_{L^{q}}, \quad 1 \leq q \leq \infty .
$$

where $C_{d}$ is a constant depending on $d$ only.

Remark 2.4. This proposition is invalid in the case when $N=-1$. The proof requires the symbol of $\Delta_{N}$ is supported away from the origin.

Proof of Proposition 2.3. Clearly,

$$
\left(\Delta_{N} \nabla u\right)_{j k}=\mathcal{R}_{l} \mathcal{R}_{m} P(\Lambda) \Delta_{N} \theta
$$

and

$$
\left(\widehat{\Delta_{N} \nabla u}\right)_{j k}(\xi)=-\frac{\xi_{l} \xi_{m}}{|\xi|^{2}} P(|\xi|) \widehat{\Delta_{N} \theta}(\xi) .
$$

As defined in Appendix A, $\widehat{\Delta_{N} \theta}(\xi)=\phi\left(\xi / 2^{N}\right) \widehat{\theta}(\xi)$ with $\phi\left(\xi / 2^{N}\right)$ supported in the annulus $(3 / 4) 2^{N} \leq|\xi| \leq(11 / 6) 2^{N}$. It is not hard to see that there exists a smooth radial function $\widetilde{\phi}_{N}$ satisfying

$$
\widetilde{\phi}_{N} \equiv 1 \text { for }(3 / 4) 2^{N} \leq|\xi| \leq(11 / 6) 2^{N} \quad \text { and } \operatorname{supp} \widetilde{\phi}_{N} \subset\left\{\xi: 2^{N-1} \leq|\xi| \leq 2^{N+1}\right\} .
$$


Then

or

$$
\left(\widehat{\Delta_{N} \nabla u}\right)_{j k}(\xi)=-\frac{\xi_{l} \xi_{m}}{|\xi|^{2}} P(|\xi|) \widetilde{\phi}_{N}(\xi) \widehat{\Delta_{N} \theta}(\xi)
$$

$$
\left(\Delta_{N} \nabla u\right)_{j k}=g * \Delta_{N} \theta
$$

where $g$ denotes the inverse Fourier transform

$$
g(x)=\frac{1}{(2 \pi)^{d / 2}} \int_{\mathbb{R}^{d}} e^{i x \cdot \xi}\left(-\frac{\xi_{l} \xi_{m}}{|\xi|^{2}} P(|\xi|) \widetilde{\phi}_{N}(\xi)\right) d \xi
$$

Clearly, $g(x)=2^{N d} g_{1}\left(2^{N} x\right)$, where

$$
g_{1}(x)=-\frac{1}{(2 \pi)^{d / 2}} \int_{2^{-1} \leq|\eta| \leq 2} 2^{i x \cdot \eta} \frac{\eta_{l} \eta_{m}}{|\eta|^{2}} P\left(2^{N}|\eta|\right) \widetilde{\phi}_{0}(\eta) d \eta
$$

with $\widetilde{\phi}_{0}(\eta)=\widetilde{\phi}_{N}\left(2^{N} \eta\right)$. To show $g \in L^{1}\left(\mathbb{R}^{d}\right)$, it suffices to show $g_{1} \in L^{1}\left(\mathbb{R}^{d}\right)$. Since

$$
\left(1+|x|^{2}\right)^{n} g_{1}(x)=-\frac{1}{(2 \pi)^{d / 2}} \int_{2^{-1} \leq|\eta| \leq 2} 2^{i x \cdot \eta}\left(I-\Delta_{\eta}\right)^{n} \frac{\eta_{l} \eta_{m}}{|\eta|^{2}} P\left(2^{N}|\eta|\right) \widetilde{\phi}_{0}(\eta) d \eta,
$$

we obtain, by (4) in Assumption 1.1,

$$
\left(1+|x|^{2}\right)^{n}\left|g_{1}(x)\right| \leq C P\left(C_{0} 2^{N}\right) .
$$

where $C$ is constant independent of $N$. (2.4) then follows from Young's inequality.

We now prove (1.8) of Theorem 1.2. In fact, we have the following proposition.

Proposition 2.5. Let $u: \mathbb{R}^{d} \rightarrow \mathbb{R}^{d}$ be a vector field. Assume that $u$ is related to a scalar $\theta$ by

$$
(\nabla u)_{j k}=\mathcal{R}_{l} \mathcal{R}_{m} P(\Lambda) \theta
$$

where $1 \leq j, k, l, m \leq d,(\nabla u)_{j k}$ denotes the $(j, k)$-th entry of $\nabla u$ and $\mathcal{R}_{l}$ denotes the Riesz transform. Here $P$ obeys Assumption 1.1. Then, for any integer $N \geq 0$,

$$
\left\|S_{N} \nabla u\right\|_{L^{\infty}} \leq C_{d}\|\theta\|_{L^{1} \cap L^{\infty}}+C_{d} N P\left(C_{0} 2^{N}\right)\left\|S_{N+1} \theta\right\|_{L^{\infty}},
$$

where $C_{d}$ depends on $d$ only.

Proof. Splitting $S_{N}$ into two parts and applying Proposition 2.3 with $q=\infty$, we have

$$
\begin{aligned}
\left\|\nabla S_{N} u\right\|_{L^{\infty}} & \leq\left\|\nabla \Delta_{-1} u\right\|_{L^{\infty}}+\sum_{j=0}^{N-1}\left\|\nabla \Delta_{j} u\right\|_{L^{\infty}} \\
& \leq C_{d}\left\|\Delta_{-1} \theta\right\|_{L^{2}}+\sum_{j=0}^{N-1} C_{d} P\left(C_{0} 2^{j}\right)\left\|\Delta_{j} \theta\right\|_{L^{\infty}}
\end{aligned}
$$

Since $P$ is nondecreasing according to Assumption 1.1 and the simple fact that

$$
\left\|\Delta_{j} \theta\right\|_{L^{\infty}} \leq\left\|S_{N+1} \theta\right\|_{L^{\infty}}, \quad j=0,1, \cdots, N-1,
$$

we have

$$
\left\|\nabla S_{N} u\right\|_{L^{\infty}} \leq C_{d}\|\theta\|_{L^{1} \cap L^{\infty}}+C_{d} N P\left(C_{0} 2^{N}\right)\left\|S_{N+1} \theta\right\|_{L^{\infty}},
$$

which is (2.5). 
We now prove Proposition 1.4, in which $P$ assumes the special form

$$
P(\Lambda)=(\log (I+\log (I-\Delta)))^{\gamma} .
$$

Proof of Proposition 1.4. For any integer $N \geq 0$, we have

$$
\|\nabla u\|_{L^{\infty}} \leq\left\|\Delta_{-1} \nabla u\right\|_{L^{\infty}}+\sum_{k=0}^{N-1}\left\|\Delta_{k} \nabla u\right\|_{L^{\infty}}+\sum_{k=N}^{\infty}\left\|\Delta_{k} \nabla u\right\|_{L^{\infty}} .
$$

By Bernstein's inequality and Proposition 2.3 , we have

$$
\begin{aligned}
\|\nabla u\|_{L^{\infty}} \leq & C_{d}\|\theta\|_{L^{1} \cap L^{\infty}}+C_{d} N\left(\log \left(1+\log \left(1+2^{2(N-1)}\right)\right)\right)^{\gamma}\|\theta\|_{L^{\infty}} \\
& +C_{d} \sum_{k=N}^{\infty}\left(2^{k}\right)^{\frac{d}{q}}\left\|\nabla \Delta_{k} u\right\|_{L^{q}} .
\end{aligned}
$$

Since $\log \left(1+2^{2(N-1)}\right)=\left(\log _{2} e\right)^{-1} \log _{2}\left(1+2^{2(N-1)}\right) \leq 2 N$, we apply Proposition 2.3 again to obtain

$$
\begin{aligned}
\|\nabla u\|_{L^{\infty}} \leq & C_{d}\|\theta\|_{L^{1} \cap L^{\infty}}+C_{d} N(\log (1+N))^{\gamma}\|\theta\|_{L^{\infty}} \\
& +C_{d} \sum_{k=N}^{\infty}\left(2^{k}\right)^{\frac{d}{q}}(\log (1+k))^{\gamma}\left\|\Delta_{k} \theta\right\|_{L^{q} .}
\end{aligned}
$$

By the definition of Besov space $B_{q, \infty}^{s}$ (see Appendix $\underline{\text { A }}$ ),

$$
\left\|\Delta_{k} \theta\right\|_{L^{q}} \leq 2^{-s k}\|\theta\|_{B_{q, \infty}^{s}}
$$

Therefore,

$$
\begin{aligned}
\|\nabla u\|_{L^{\infty}} \leq & C_{d}\|\theta\|_{L^{1} \cap L^{\infty}}+C_{d} N(\log (1+N))^{\gamma}\|\theta\|_{L^{\infty}} \\
& +C_{d}\|\theta\|_{B_{q, \infty}^{s}} \sum_{k=N}^{\infty}\left(2^{k}\right)^{\left(\frac{d}{q}-s\right)}(\log (1+k))^{\gamma} .
\end{aligned}
$$

Since $d / q-s<0$, we obtain for large $N$,

$$
\begin{aligned}
\|\nabla u\|_{L^{\infty}} \leq & C_{d}\|\theta\|_{L^{1} \cap L^{\infty}}+C_{d} N(\log (1+N))^{\gamma}\|\theta\|_{L^{\infty}} \\
& +C_{d, q, s}\|\theta\|_{B_{q, \infty}^{s}}\left(2^{N}\right)^{\left(\frac{d}{q}-s\right)}(\log (1+N))^{\gamma} .
\end{aligned}
$$

If we choose $N$ to be the largest integer satisfying

$$
N \leq \frac{1}{s-d / q} \log \left(1+\|\theta\|_{B_{q, \infty}^{s}}\right)
$$

we then obtain the desired result in Proposition 1.4.

\section{Global Regularity For (1.1) With $P(\Lambda)=(\log (1+\log (1-\Delta)))^{\gamma}$}

This section establishes the global existence and uniqueness of solutions to (1.1) with $P(\Lambda)=(\log (1+\log (1-\Delta)))^{\gamma}$. The divergence-free condition on the velocity field $u$ is not necessary if we are willing to assume that $\theta$ is bounded in $L^{1} \cap L^{\infty}$ for all time. Of course when $u$ is indeed divergence-free, the bound is then a trivial consequence. In the $2 \mathrm{D}$ case, this general theorem reduces to Theorem 1.3 stated in the introduction. 
Theorem 3.1. Consider the active scalar equation (1.1) with

$$
P(\Lambda)=(\log (1+\log (1-\Delta)))^{\gamma}, \quad 0 \leq \gamma \leq 1 .
$$

Assume that the initial data $\theta_{0}$ satisfies

$$
\theta_{0} \in X \equiv L^{1}\left(\mathbb{R}^{d}\right) \cap L^{\infty}\left(\mathbb{R}^{d}\right) \cap B_{q, \infty}^{s}\left(\mathbb{R}^{d}\right),
$$

with

$$
d<q \leq \infty \text { and } s>1 .
$$

Assume either $u$ is divergence-free or $\theta$ is bounded in $L^{1} \cap L^{\infty}$ for all time. Then (1.1) has a unique global in time solution $\theta$ that satisfies

$$
\theta \in L^{\infty}\left([0, \infty) ; B_{q, \infty}^{s}\left(\mathbb{R}^{d}\right)\right) \quad \text { and } \quad u \in L^{\infty}\left([0, \infty) ; B_{q, \infty}^{1+s^{\prime}}\left(\mathbb{R}^{d}\right)\right)
$$

for any $s^{\prime}<s$.

Proof. The proof consists of two main components. The first component derives a global a priori bound while the second constructs a unique local in time solution through the method of successive approximation.

We start with the part on the global a priori bound. This part is further divided into two steps. The first step shows that for any $d / q<\sigma<1$ and any $T>0$,

$$
\|\theta(t)\|_{B_{q, \infty}^{\sigma}} \leq C\left(T,\left\|\theta_{0}\right\|_{X}\right), \quad t \leq T
$$

and the second step establishes the global bound in $B_{q, \infty}^{\sigma_{1}}$ for some $\sigma_{1}>1$. A finite number of iterations then yields the global bound in $B_{q, \infty}^{s}$.

When $u$ is divergence-free, $\theta_{0} \in L^{1} \cap L^{\infty}$ implies that the corresponding solution $\theta$ of (1.9) satisfies the a priori bound

$$
\|\theta(\cdot, t)\|_{L^{1} \cap L^{\infty}} \leq\left\|\theta_{0}\right\|_{L^{1} \cap L^{\infty}}, \quad t \geq 0 .
$$

When $u$ is not divergence-free, we assume that (3.1) holds. Of course, the bound does not have to be $\left\|\theta_{0}\right\|_{L^{1} \cap L^{\infty}}$. In the rest of the proof, we can completely avoid using the divergence-free condition on $u$. This explains why the divergence-free condition is not used in the estimates.

Let $j \geq-1$ be an integer. Applying $\Delta_{j}$ to (1.9) and following a standard decomposition, we have

$$
\partial_{t} \Delta_{j} \theta=J_{1}+J_{2}+J_{3}+J_{4}+J_{5}
$$


where

$$
\begin{aligned}
& J_{1}=-\sum_{|j-k| \leq 2}\left[\Delta_{j}, S_{k-1}(u) \cdot \nabla\right] \Delta_{k} \theta, \\
& J_{2}=-\sum_{|j-k| \leq 2}\left(S_{k-1}(u)-S_{j}(u)\right) \cdot \nabla \Delta_{j} \Delta_{k} \theta, \\
& J_{3}=-S_{j}(u) \cdot \nabla \Delta_{j} \theta \\
& J_{4}=-\sum_{|j-k| \leq 2} \Delta_{j}\left(\Delta_{k} u \cdot \nabla S_{k-1}(\theta)\right), \\
& J_{5}=-\sum_{k \geq j-1} \Delta_{j}\left(\Delta_{k} u \cdot \nabla \widetilde{\Delta}_{k} \theta\right)
\end{aligned}
$$

with $\widetilde{\Delta}_{k}=\Delta_{k-1}+\Delta_{k}+\Delta_{k+1}$. Multiplying (3.2) by $\Delta_{j} \theta\left|\Delta_{j} \theta\right|^{q-2}$, integrating in space, integrating by part in the term associated with $J_{3}$, and applying Hölder's inequality, we have

$$
\frac{d}{d t}\left\|\Delta_{j} \theta\right\|_{L^{q}} \leq\left\|J_{1}\right\|_{L^{q}}+\left\|J_{2}\right\|_{L^{q}}+\left\|\widetilde{J}_{3}\right\|_{L^{q}}+\left\|J_{4}\right\|_{L^{q}}+\left\|J_{5}\right\|_{L^{q}}
$$

By a standard commutator estimate,

$$
\left\|J_{1}\right\|_{L^{q}} \leq C \sum_{|j-k| \leq 2}\left\|\nabla S_{k-1} u\right\|_{L^{\infty}}\left\|\Delta_{k} \theta\right\|_{L^{q}}
$$

By Hölder's and Bernstein's inequalities,

$$
\left\|J_{2}\right\|_{L^{q}} \leq C\left\|\nabla \widetilde{\Delta}_{j} u\right\|_{L^{\infty}}\left\|\Delta_{j} \theta\right\|_{L^{q}} .
$$

After integration by parts, the term $J_{3}$ leads to a term $\widetilde{J}_{3}=\frac{1}{q}\left(\nabla \cdot S_{j} u\right) \Delta_{j} \theta$, and so

$$
\left\|\widetilde{J}_{3}\right\|_{L^{q}} \leq C\left\|\nabla \cdot S_{j} u\right\|_{L^{\infty}}\left\|\Delta_{j} \theta\right\|_{L^{q}} .
$$

For $J_{4}$ and $J_{5}$, we have

$$
\begin{aligned}
\left\|J_{4}\right\|_{L^{q}} & \leq \sum_{|j-k| \leq 2}\left\|\Delta_{k} u\right\|_{L^{\infty}}\left\|\nabla S_{k-1} \theta\right\|_{L^{q}} \\
& \leq C \sum_{|j-k| \leq 2}\left\|\nabla \Delta_{k} u\right\|_{L^{\infty}} \sum_{m \leq k-1} 2^{m-k}\left\|\Delta_{m} \theta\right\|_{L^{q}} \\
\left\|J_{5}\right\|_{L^{q}} & \leq C \sum_{k \geq j-1}\left\|\Delta_{k} u\right\|_{L^{\infty}}\left\|\widetilde{\Delta}_{k} \nabla \theta\right\|_{L^{q}} \\
& \leq C \sum_{k \geq j-1}\left\|\nabla \Delta_{k} u\right\|_{L^{\infty}}\left\|\widetilde{\Delta}_{k} \theta\right\|_{L^{q}}
\end{aligned}
$$

By Proposition 1.4, for any $\sigma \in \mathbb{R}$,

$$
\begin{aligned}
\left\|J_{1}\right\|_{L^{q}} & \leq C \sum_{|j-k| \leq 2}\|\nabla u\|_{L^{\infty}} 2^{-\sigma(k+1)} 2^{\sigma(k+1)}\left\|\Delta_{k} \theta\right\|_{L^{q}} \\
& \leq C 2^{-\sigma(j+1)}\|\theta\|_{B_{q, \infty}^{\sigma}}\|\nabla u\|_{L^{\infty}} \sum_{|j-k| \leq 2} 2^{\sigma(j-k)} \\
& \leq C 2^{-\sigma(j+1)}\|\theta\|_{B_{q, \infty}^{\sigma}}\|\nabla u\|_{L^{\infty}}
\end{aligned}
$$


where $C$ is a constant depending on $\sigma$ only. It is clear that $\left\|J_{2}\right\|_{L^{q}}$ and $\left\|\widetilde{J}_{3}\right\|_{L^{q}}$ obey the same bound. For any $\sigma<1$, we have

$$
\begin{aligned}
\left\|J_{4}\right\|_{L^{q}} & \leq C\|\nabla u\|_{L^{\infty}} \sum_{|j-k| \leq 2} \sum_{m<k-1} 2^{m-k} 2^{-\sigma(m+1)} 2^{\sigma(m+1)}\left\|\Delta_{m} \theta\right\|_{L^{q}} \\
& \leq C\|\nabla u\|_{L^{\infty}}\|\theta\|_{B_{q, \infty}^{\sigma}} \sum_{|j-k| \leq 2} \sum_{m<k-1} 2^{m-k} 2^{-\sigma(m+1)} \\
& =C 2^{-\sigma(j+1)}\|\theta\|_{B_{q, \infty}^{\sigma}}\|\nabla u\|_{L^{\infty}} \sum_{|j-k| \leq 2} 2^{\sigma(j-k)} \sum_{m<k-1} 2^{(m-k)(1-\sigma)} \\
& \leq C 2^{-\sigma(j+1)}\|\theta\|_{B_{q, \infty}^{\sigma}}\|\nabla u\|_{L^{\infty}} .
\end{aligned}
$$

where $C$ is a constant depending on $\sigma$ only and the condition $\sigma<1$ is used to guarantee that $(m-k)(1-\sigma)<0$. For any $\sigma>0$,

$$
\begin{aligned}
\left\|J_{5}\right\|_{L^{q}} & \leq C\|\nabla u\|_{L^{\infty}} 2^{-\sigma(j+1)} \sum_{k \geq j-1} 2^{\sigma(j-k)} 2^{\sigma(k+1)}\left\|\widetilde{\Delta}_{k} \theta\right\|_{L^{q}} \\
& \leq C 2^{-\sigma(j+1)}\|\theta\|_{B_{q, \infty}^{\sigma}}\|\nabla u\|_{L^{\infty}} .
\end{aligned}
$$

Collecting these estimates, we obtain, for any $0<\sigma<1$,

$$
\frac{d}{d t}\left\|\Delta_{j} \theta\right\|_{L^{q}} \leq C 2^{-\sigma(j+1)}\|\theta\|_{B_{q, \infty}^{\sigma}}\|\nabla u\|_{L^{\infty}} .
$$

Integrating in time yields

$$
\|\theta(t)\|_{B_{q, \infty}^{\sigma}} \leq\left\|\theta_{0}\right\|_{B_{q, \infty}^{\sigma}}+C \int_{0}^{t}\|\theta(\tau)\|_{B_{q, \infty}^{\sigma}}\|\nabla u(\tau)\|_{L^{\infty}} d \tau
$$

Invoking the extrapolation inequality in Proposition 1.4, we obtain, for $d / q<\sigma<1$,

$$
\begin{aligned}
\|\theta(t)\|_{B_{q, \infty}^{\sigma}} \leq\left\|\theta_{0}\right\|_{B_{q, \infty}^{\sigma}}+C \int_{0}^{t}\|\theta(\tau)\|_{B_{q, \infty}^{\sigma}}\left[\|\theta\|_{L^{1} \cap L^{\infty}}+\left(1+\|\theta\|_{L^{\infty}}\right)\right. \\
\left.\times \log \left(1+\|\theta\|_{B_{q, \infty}^{\sigma}}\right)\left(\log \left(1+\log \left(1+\|\theta\|_{B_{q, \infty}^{\sigma}}\right)\right)\right)^{\gamma}\right] d \tau .
\end{aligned}
$$

It then follows from Gronwall's inequality that, for any $T>0$,

$$
\|\theta(t)\|_{B_{q, \infty}^{\sigma}} \leq C\left(T,\left\|\theta_{0}\right\|_{X}\right), \quad t \leq T .
$$

We now continue with the second step. Since $d<q \leq \infty$, we can choose $\sigma$ satisfying

$$
\frac{d}{q}<\sigma<1, \quad \sigma+1-\frac{d}{q}>1
$$

and then set $\sigma_{1}$ satisfying

$$
1<\sigma_{1}<\sigma+1-\frac{d}{q}
$$

This step establishes the global bound for $\|\theta\|_{B_{q, \infty}^{\sigma_{1}}} . J_{1}, J_{2}$ and $J_{3}$ and $J_{5}$ can be bounded the same way as before, namely

$$
\left\|J_{1}\right\|_{L^{q}},\left\|J_{2}\right\|_{L^{q}},\left\|\widetilde{J}_{3}\right\|_{L^{q}},\left\|J_{5}\right\|_{L^{q}} \leq C 2^{-\sigma_{1}(j+1)}\|\theta\|_{B_{q, \infty}^{\sigma_{1}}}\|\nabla u\|_{L^{\infty}}
$$


$\left\|J_{4}\right\|_{L^{q}}$ is estimated differently and bounded by the global bound in the first step. We start with the bound

$$
\left\|J_{4}\right\|_{L^{q}} \leq C \sum_{|j-k| \leq 2}\left\|\nabla \Delta_{k} u\right\|_{L^{\infty}} \sum_{m<k-1} 2^{m-k}\left\|\Delta_{m} \theta\right\|_{L^{q}} .
$$

By Bernstein's inequality and Proposition 2.3, we have

$$
\begin{aligned}
\left\|\nabla \Delta_{k} u\right\|_{L^{\infty}} & \leq 2^{\frac{d k}{q}}\left\|\nabla \Delta_{k} u\right\|_{L^{q}} \\
& \leq 2^{\frac{d k}{q}}(\log (2+k))^{\gamma}\left\|\Delta_{k} \theta\right\|_{L^{q}} .
\end{aligned}
$$

Clearly,

$$
\begin{aligned}
\sum_{m<k-1} 2^{m-k}\left\|\Delta_{m} \theta\right\|_{L^{q}} & =2^{-\sigma k} \sum_{m<k-1} 2^{(m-k)(1-\sigma)} 2^{\sigma m}\left\|\Delta_{m} \theta\right\|_{L^{q}} \\
& \leq C 2^{-\sigma k}\|\theta\|_{B_{q, \infty}^{\sigma}} .
\end{aligned}
$$

Therefore,

$$
\begin{aligned}
\left\|J_{4}\right\|_{L^{q}} & \leq C \sum_{|j-k| \leq 2} 2^{\frac{d k}{q}}(\log (2+k))^{\gamma}\left\|\Delta_{k} \theta\right\|_{L^{q}} 2^{-\sigma k}\|\theta\|_{B_{q, \infty}^{\sigma}} \\
& =C 2^{-\sigma_{1}(j+1)}\|\theta\|_{B_{q, \infty}^{\sigma}} \sum_{|j-k| \leq 2} 2^{\sigma_{1}(j-k)}(\log (2+k))^{\gamma} 2^{\left(\sigma_{1}+\frac{d}{q}-\sigma\right) k}\left\|\Delta_{k} \theta\right\|_{L^{q}} \\
& =C 2^{-\sigma_{1}(j+1)}\|\theta\|_{B_{q, \infty}^{\sigma}}\|\theta\|_{B_{q, \infty}^{\sigma_{2}}} \sum_{|j-k| \leq 2} 2^{\sigma_{1}(j-k)}(\log (2+k))^{\gamma} 2^{\left(\sigma_{1}+\frac{d}{q}-\sigma-\sigma_{2}\right) k}
\end{aligned}
$$

where $\sigma_{2}<1$ is chosen very close to 1 and satisfies

$$
\sigma_{1}+\frac{2}{q}-\sigma-\sigma_{2}<0
$$

Then, by the global bound in the first step,

$$
\left\|J_{4}\right\|_{L^{q}} \leq C 2^{-\sigma_{1}(j+1)}\|\theta\|_{B_{q, \infty}^{\sigma}}\|\theta\|_{B_{q, \infty}^{\sigma_{2}}} \leq C\left(T,\left\|\theta_{0}\right\|_{X}\right) 2^{-\sigma_{1}(j+1)} .
$$

Collecting the estimates in this step, we have

$$
\frac{d}{d t}\left\|\Delta_{j} \theta\right\|_{L^{q}} \leq C 2^{-\sigma_{1}(j+1)}\|\theta\|_{B_{q, \infty}^{\sigma_{1}}}\|\nabla u\|_{L^{\infty}}+C\left(T,\left\|\theta_{0}\right\|_{X}\right) 2^{-\sigma_{1}(j+1)} .
$$

By Proposition 1.4, for any $d / q<\sigma<1$,

$$
\begin{aligned}
\|\nabla u\|_{L^{\infty}} \leq & \|\theta\|_{L^{1} \cap L^{\infty}}+\left(1+\|\theta\|_{L^{\infty}}\right) \\
& \quad \times \log \left(1+\|\theta\|_{B_{q, \infty}^{\sigma}}\right)\left(\log \left(1+\log \left(1+\|\theta\|_{B_{q, \infty}^{\sigma}}\right)\right)\right)^{\gamma} \\
\leq & C\left(T,\left\|\theta_{0}\right\|_{X}\right) .
\end{aligned}
$$

Therefore,

$$
\|\theta(t)\|_{B_{q, \infty}^{\sigma_{1}}} \leq\left\|\theta_{0}\right\|_{B_{q, \infty}^{\sigma_{1}}}+C\left(T,\left\|\theta_{0}\right\|_{X}\right)\left(1+\int_{0}^{t}\|\theta(\tau)\|_{B_{q, \infty}^{\sigma_{1}}} d \tau\right) .
$$

Gronwall's inequality then yields the global bound $\|\theta(t)\|_{B_{q, \infty}^{\sigma_{1}}} \leq C\left(T,\left\|\theta_{0}\right\|_{X}\right)$. If $s>\sigma_{1}$, we can repeat this step to achieve the desired regularity. 
We now describe the process of constructing a local solution of (1.1). The solution is constructed through the method of successive approximation. Consider a successive approximation sequence $\left\{\theta^{(n)}\right\}$ satisfying

$$
\left\{\begin{array}{l}
\theta^{(1)}=S_{2} \theta_{0} \\
u^{(n)}=\left(u_{j}^{(n)}\right), \quad u_{j}^{(n)}=\mathcal{R}_{l} \Lambda^{-1} P(\Lambda) \theta^{(n)} \\
\partial_{t} \theta^{(n+1)}+u^{(n)} \cdot \nabla \theta^{(n+1)}=0 \\
\theta^{(n+1)}(x, 0)=S_{n+2} \theta_{0}
\end{array}\right.
$$

where $P(\Lambda)=(\log (1+\log (1-\Delta)))^{\gamma}$. In order to show that $\left\{\theta^{(n)}\right\}$ converges to a solution of (1.1), it suffices to prove the following properties of $\left\{\theta^{(n)}\right\}$ :

(1) There exists $T_{1}>0$ such that $\theta^{(n)}$ is bounded uniformly in $B_{q, \infty}^{s}$ for any $t \in[0, T]$, namely

$$
\left\|\theta^{(n)}(\cdot, t)\right\|_{B_{q, \infty}^{s}} \leq C_{1}\left\|\theta_{0}\right\|_{X}, \quad t \in\left[0, T_{1}\right]
$$

where $C_{1}$ is a constant independent of $n$.

(2) There exists $T_{2}>0$ such that $\eta^{(n+1)}=\theta^{(n+1)}-\theta^{(n)}$ is a Cauchy sequence in $B_{q, \infty}^{s-1}$,

$$
\left\|\eta^{(n)}(\cdot, t)\right\|_{B_{q, \infty}^{s-1}} \leq C_{2} 2^{-n}, \quad t \in\left[0, T_{2}\right],
$$

where $C_{2}$ is independent of $n$ and depends on $T_{2}$ and $\left\|\theta_{0}\right\|_{X}$ only.

These two properties are established by following the ideas of the previous part and we omit the details. Let $T=\min \left\{T_{1}, T_{2}\right\}$. We conclude from these two properties that there exists $\theta$ satisfying

$$
\begin{aligned}
& \theta(\cdot, t) \in B_{q, \infty}^{s} \quad \text { for } \quad 0 \leq t \leq T, \\
& \theta^{(n)}(\cdot, t) \rightarrow \theta(\cdot, t) \quad \text { in } \quad B_{q, \infty}^{s}, \\
& \theta^{(n)}(\cdot, t) \rightarrow \theta(\cdot, t) \quad \text { in } \quad B_{q, \infty}^{s-1} .
\end{aligned}
$$

Due to the interpolation inequality, for any $s-1 \leq \widetilde{s} \leq s$,

$$
\|f\|_{B_{q, \infty}^{\widetilde{s}}} \leq C\|f\|_{B_{q, \infty}^{s-1}}^{s-\widetilde{s}}\|f\|_{B_{q, \infty}^{s}}^{\widetilde{s}+1-s},
$$

we deduce that

$$
\theta^{(n)}(\cdot, t) \rightarrow \theta(\cdot, t) \quad \text { in } \quad B_{q, \infty}^{\widetilde{s}}
$$

In addition, by the relation $u_{k}^{(n)}=\mathcal{R}_{l} \Lambda^{-1} P(\Lambda) \theta^{(n)}$ and Proposition 2.3, we can easily check that

$$
\nabla u^{(n)}, \nabla u(\cdot, t) \in B_{q, \infty}^{s_{1}} \quad \text { for any } \quad s_{1}<s .
$$

In order to pass the limit in the nonlinear term, we write

$$
u^{(n)} \cdot \nabla \theta^{(n+1)}-u \cdot \nabla \theta=u^{(n)} \cdot \nabla\left(\theta^{(n+1)}-\theta\right)+\left(u^{(n)}-u\right) \cdot \nabla \theta .
$$

We can show that, for any $\sigma<s-1$,

$$
u^{(n)} \cdot \nabla\left(\theta^{(n+1)}-\theta\right) \rightarrow 0, \quad\left(u^{(n)}-u\right) \cdot \nabla \theta \rightarrow 0 \quad \text { in } B_{q, \infty}^{\sigma},
$$

as $n \rightarrow \infty$. Again they can be proven by following the ideas in the first part of this proof. Finally the uniqueness can be established by estimating the difference of any two 
solutions in $B_{q, \infty}^{s-1}$. A similar argument as in the proof of $\left\|\eta^{(n)}(\cdot, t)\right\|_{B_{q, \infty}^{s-1}} \leq C_{2} 2^{-n}$ yields the conclusion that the difference must be zero. This completes the proof of Theorem 3.1 .

\section{Generalized Inviscid SQG EQUation}

This section is devoted to the generalized inviscid SQG equation

$$
\left\{\begin{array}{l}
\partial_{t} \theta+(u \cdot \nabla) \theta=0, \quad x \in \mathbb{R}^{2}, t>0, \\
u=\nabla^{\perp} \psi, \quad-\Lambda^{2-\beta} \psi=\theta, \quad x \in \mathbb{R}^{2}, t>0,
\end{array}\right.
$$

where $0 \leq \beta \leq 1$ is a parameter. (4.1) with $\beta=0$ becomes the $2 \mathrm{D}$ Euler vorticity equation while (4.1) with $\beta=1$ is the SQG equation. Except in the case when $\beta=0$, the global regularity issue for (4.1) remains open. This section presents a regularity criterion in terms of the norm of $\theta$ in the Hölder space $C^{\beta}\left(\mathbb{R}^{2}\right)$, which directly relates the regularity of $\theta$ to the parameter $\beta$. The precise conclusion has been stated in Theorem 1.5 and we reproduce it here.

Theorem 4.1. Consider (4.1) with $0 \leq \beta \leq 1$. Let $\theta$ be a solution of (4.1) corresponding to the data $\theta_{0} \in C^{\sigma}\left(\mathbb{R}^{2}\right) \cap L^{q}\left(\mathbb{R}^{2}\right)$ with $\sigma>1$ and $q>1$. Let $T>0$. If $\theta$ satisfies

$$
\int_{0}^{T}\|\theta(\cdot, t)\|_{C^{\beta}\left(\mathbb{R}^{2}\right)} d t<\infty
$$

then $\theta$ remains in $C^{\sigma}\left(\mathbb{R}^{2}\right) \cap L^{q}\left(\mathbb{R}^{2}\right)$ on the time interval $[0, T]$.

Some special consequences of this theorem are given in the following remark.

Remark 4.2. In the special case when $\beta=0$, Theorem 4.1 re-establishes the global regularity for the $2 D$ Euler equation. In the special case when $\beta=1$, 4.1) becomes the inviscid $S Q G$ equation and Theorem 4.1] reduces to a regularity criterion of [23] for the $S Q G$ equation.

To prove Theorem 4.1, we first establish two propositions. The first one bounds the back-to-labels map (the inverse map of the particle trajectory) in terms of the symmetric part of $\nabla u$. The second proposition is a logarithmic Hölder space inequality.

Let $X(a, t)$ be the particle trajectory determined by the velocity $u$, namely

$$
\left\{\begin{array}{l}
\frac{d X(a, t)}{d t}=u(X(a, t), t) \\
X(a, 0)=a
\end{array}\right.
$$

Let $A(x, t)$ be the back-to-labels map or the inverse map of $X$. Then

$$
A(X(a, t), t)=a \text { for any } a \in \mathbb{R}^{2} .
$$

Let $S$ denote the symmetric part of $\nabla u$, namely

$$
S=\frac{1}{2}\left(\nabla u+(\nabla u)^{T}\right)
$$

where $(\nabla u)^{T}$ denotes the transpose of $\nabla u$. The following proposition bounds $\nabla_{x} A$ in terms of $S$. 
Proposition 4.3. Let $u$ be a velocity field and let $S$ be the strain tensor as defined in (4.5). Let $A$ be the back-to-labels map. Then,

$$
\left\|\nabla_{x} A(\cdot, t)\right\|_{L^{\infty}} \leq \exp \left(\int_{0}^{t}\|S(\cdot, \tau)\|_{L^{\infty}} d \tau\right) .
$$

The second proposition bounds the $L^{\infty}$-norm of $S$ in terms of the logarithm of the Hölder-norm of $\theta$.

Proposition 4.4. Let $0 \leq \beta \leq 1$. Assume that $u$ and $\theta$ are related by

$$
u=-\nabla^{\perp} \Lambda^{-2+\beta} \theta
$$

If $\theta \in C^{\sigma}\left(\mathbb{R}^{2}\right) \cap L^{q}\left(\mathbb{R}^{2}\right)$ with $\sigma>\beta$ and $q>1$,

$$
\|S\|_{L^{\infty}} \leq C_{1}\|\theta\|_{C^{\beta}} \ln \left(1+\|\theta\|_{C^{\sigma}}\right)+C_{2}\|\theta\|_{L^{q}}
$$

where $C_{1}$ and $C_{2}$ are constants depending on $\beta, \sigma$ and $q$ only.

The rest of this section is arranged as follows. We prove Theorem 4.1 first and then provide the proofs of Propositions 4.3 and 4.4 .

Proof of Theorem 4.1. Let $X$ be the particle trajectory as defined in (4.3) and $A(x, t)$ be the back-to-labels map. The first equation in (4.1) implies that $\theta$ is conserved along the particle trajectory,

$$
\theta(x, t)=\theta_{0}(A(x, t)), \quad x \in \mathbb{R}^{2}, t \geq 0 .
$$

Therefore, for any $\sigma \leq 1$,

$$
\|\theta(\cdot, t)\|_{C^{\sigma}}=\sup _{x \neq y} \frac{|\theta(x, t)-\theta(y, t)|}{|x-y|^{\sigma}} \leq\left\|\theta_{0}\right\|_{C^{\sigma}}\left\|\nabla_{x} A(\cdot, t)\right\|_{L^{\infty}}^{\sigma} .
$$

By Proposition 4.3, we have

$$
\|\theta(\cdot, t)\|_{C^{\sigma}} \leq\left\|\theta_{0}\right\|_{C^{\sigma}} \exp \left(\sigma \int_{0}^{t}\|S(\cdot, \tau)\|_{L^{\infty}} d \tau\right) .
$$

Therefore,

$$
\ln \left(1+\|\theta(\cdot, t)\|_{C^{\sigma}}\right) \leq \ln \left(1+\left\|\theta_{0}\right\|_{C^{\sigma}}\right)+\sigma \int_{0}^{t}\|S(\cdot, \tau)\|_{L^{\infty}} d \tau .
$$

According to Proposition 4.4,

$$
\int_{0}^{t}\|S(\cdot, \tau)\|_{L^{\infty}} d \tau \leq C_{1} \int_{0}^{t}\|\theta(\cdot, \tau)\|_{C^{\beta}} \ln \left(1+\|\theta(\cdot, \tau)\|_{C^{\sigma}}\right) d \tau+C_{2} t\left\|\theta_{0}\right\|_{L^{q}}
$$

Combining (4.8) and (4.9) and applying Gronwall's inequality yield

$$
\ln \left(1+\|\theta(\cdot, t)\|_{C^{\sigma}}\right) \leq C \ln \left(1+\left\|\theta_{0}\right\|_{C^{\sigma}}+\left\|\theta_{0}\right\|_{L^{q}}\right) \exp \left(C \int_{0}^{t}\|\theta(\cdot, \tau)\|_{C^{\beta}} d \tau\right) .
$$

In particular, taking $\sigma=1$ yields a bound for $\|\nabla \theta\|_{L^{\infty}}$. The desired regularity $\theta \in C^{\sigma}$ with $\sigma>1$ then follows easily from the bound for $\|\nabla \theta\|_{L^{\infty}}$. This completes the proof of Theorem 4.1. 
Proof of Proposition 4.3. Differentiating the identity in (4.4) with respect to $t$, we obtain the equation for $A$,

$$
\partial_{t} A+u \cdot \nabla A=0 .
$$

Taking the gradient with respect to $x$, we find

$$
\partial_{t}\left(\nabla_{x} A\right)+u \cdot \nabla\left(\nabla_{x} A\right)=\nabla u\left(\nabla_{x} A\right) .
$$

Taking (Euclidian) inner product of this equation with $\nabla_{x} A$, we find

$$
\left.\frac{1}{2} \frac{D}{D t}\left|\nabla_{x} A(x, t)\right|^{2}=-\nabla u\left(\nabla_{x} A\right)\right) \cdot\left(\nabla_{x} A\right) .
$$

Adopting the Einstein summation convention, we have

$$
\left(\nabla u\left(\nabla_{x} A\right)\right) \cdot\left(\nabla_{x} A\right)=\partial_{x_{k}} u_{j} \partial_{x_{j}} A_{i} \partial_{x_{k}} A_{i}=\partial_{x_{j}} u_{k} \partial_{x_{k}} A_{i} \partial_{x_{j}} A_{i}
$$

and thus

$$
\left(\nabla u\left(\nabla_{x} A\right)\right) \cdot\left(\nabla_{x} A\right)=\left((\nabla u)^{T}\left(\nabla_{x} A\right)\right) \cdot\left(\nabla_{x} A\right)=\left(S\left(\nabla_{x} A\right)\right) \cdot\left(\nabla_{x} A\right) .
$$

Therefore

$$
\frac{1}{2} \frac{D}{D t}\left|\nabla_{x} A\right|^{2} \leq\left.\left|S(x, t)\left\|\left.\nabla_{x} A\right|^{2} \leq\right\| S(\cdot, t) \|_{L^{\infty}}\right| \nabla_{x} A\right|^{2}
$$

and integrating along the particle trajectory we obtain

$$
\left|\nabla_{x} A(X(a, t), t)\right| \leq \exp \left(\int_{0}^{t}\|S(\cdot, \tau)\|_{L^{\infty}} d \tau\right) .
$$

Proposition 4.3 follows from this immediately, taking supremum over $a \in \mathbb{R}^{2}$.

Proof of Proposition 4.4. The proof is divided into two cases: $\beta<1$ and $\beta=1$. The case $\beta=1$ requires that $\sigma>1$ and is handled differently from the case $\beta<1$.

We first deal with the case when $\beta<1$. Invoking the Riesz potential for the operator $\Lambda^{-2+\beta}$, the relation in (4.6) can be rewritten

$$
u(x)=C_{\beta} \int \nabla^{\perp}\left(\frac{1}{|x-y|^{\beta}}\right) \theta(y) d y=\int K_{\beta}(x-y) \theta(y) d y
$$

with

$$
K_{\beta}(x)=C_{\beta} \frac{\left(-x_{2}, x_{1}\right)^{T}}{|x|^{2+\beta}}
$$

where $C_{\beta}$ is a constant depending on $\beta$ only. $\nabla u$ can be written as

$$
\nabla u(x)=\text { p.v. } \int \nabla_{x} K(x-y) \theta(y) d y
$$

where p.v. denotes the principal value and $\nabla_{x} K(x)$ can be explicitly written as

$$
\nabla_{x} K(x)=C_{\beta} \frac{1}{|x|^{4+\beta}}\left(\begin{array}{cc}
x_{1} x_{2} & x_{2}^{2} \\
-x_{1}^{2} & -x_{1} x_{2}
\end{array}\right)+C_{\beta} \frac{1}{|x|^{2+\beta}}\left(\begin{array}{cc}
0 & -1 \\
1 & 0
\end{array}\right) .
$$

Therefore the symmetric part of $\nabla u$ can be written as

$$
S(x)=\text { p.v. } \int \Gamma(x-y) \theta(y) d y
$$


where

$$
\Gamma(x)=C_{\beta} \frac{1}{|x|^{4+\beta}}\left(\begin{array}{cc}
2 x_{1} x_{2} & x_{2}^{2}-x_{1}^{2} \\
x_{2}^{2}-x_{1}^{2} & -2 x_{1} x_{2}
\end{array}\right) .
$$

The property that $\Gamma(x)$ is homogenous of degree $-(2+\beta)$ and has zero mean on the unit circle is useful in the following estimate of $S$.

Let $\chi(x)$ be a standard smooth cutoff function with $\chi(x)=1$ for $|x| \leq \frac{1}{2}$ and $\chi(x)=0$ for $|x| \geq 1$. Let $0<\rho \leq R$. We divide $S$ into three parts,

$$
S(x, t)=L_{1}+L_{2}+L_{3},
$$

where

$$
\begin{aligned}
L_{1} & =\int \chi\left(\frac{|x-y|}{\rho}\right) \Gamma(x-y)(\theta(y)-\theta(x)) d y, \\
L_{2} & =\int_{|x-y| \leq R}\left(1-\chi\left(\frac{|x-y|}{\rho}\right)\right) \Gamma(x-y)(\theta(y)-\theta(x)) d y, \\
L_{3} & =\int_{|x-y|>R} \Gamma(x-y) \theta(y) d y .
\end{aligned}
$$

Since $\sigma>\beta$,

$$
\begin{aligned}
\left|L_{1}\right| & \leq C_{\beta}\|\theta\|_{C^{\sigma}} \int_{|x-y| \leq \rho} \frac{1}{|x-y|^{2+\beta-\sigma}} d y \\
& =C_{\beta}\|\theta\|_{C^{\sigma}} \rho^{\sigma-\beta} .
\end{aligned}
$$

$L_{2}$ can be bounded as follows.

$$
\begin{aligned}
\left|L_{2}\right| & \leq C_{\beta}\|\theta\|_{C^{\beta}} \int_{\frac{\rho}{2} \leq|x-y| \leq R} \frac{1}{|x-y|^{2}} d y \\
& =C_{\beta}\|\theta\|_{C^{\beta}} \ln \left(\frac{2 R}{\rho}\right) .
\end{aligned}
$$

By Hölder's inequality,

$$
\left|L_{3}\right| \leq C_{\beta, q} R^{-1-\beta}\|\theta\|_{L^{q}}
$$

Setting $\rho=\ln \left(1+\|\theta\|_{C^{\sigma}}\right)$ and $R=1$ yields (4.7).

We now turn to the case when $\beta=1$. This case corresponds to the SQG equation. Then $\sigma>\beta=1$. It follows from the relation in (4.6) that

$$
\nabla u(x)=\text { p.v. } \int \hat{y} \otimes \nabla \theta(x+y) \frac{d y}{|y|^{2}}
$$

where $\hat{y}$ denotes the unit vector in the direction of $y$ and $a \otimes b$ denotes the tensor product of two vectors $a$ and $b$. Therefore,

$$
S(x)=\text { p.v. } \int \frac{1}{2}(\hat{y} \otimes \nabla \theta(x+y)+\nabla \theta(x+y) \otimes \hat{y}) \frac{d y}{|y|^{2}} .
$$

The difference between this representation and the one in the case $\beta<1$ is that this formula involves $\nabla \theta$ instead of just $\theta$. $\|S\|_{L^{\infty}}$ can be bounded in a similar fashion as in the case $\beta<1$. In fact, we again use a smooth cutoff function $\chi$ to decompose 
the integral into three parts and estimate each one of them as we did previously. For example,

$$
L_{1}=\text { p.v. } \int \chi\left(\frac{|y|}{\rho}\right) \frac{1}{2}(\hat{y} \otimes(\nabla \theta(x+y)-\nabla \theta(x))+(\nabla \theta(x+y)-\nabla \theta(x)) \otimes \hat{y}) \frac{d y}{|y|^{2}}
$$

can be bounded by

$$
\begin{aligned}
\left|L_{1}\right| & \leq \int_{|y| \leq \rho}|\nabla \theta(x+y)-\nabla \theta(x)| \frac{d y}{|y|^{2}} \\
& \leq\|\nabla \theta\|_{C^{\sigma-1}} \rho^{\sigma-1} \leq\|\theta\|_{C^{\sigma}} \rho^{\sigma-1} .
\end{aligned}
$$

We omit details for the estimates of the other parts. Putting the estimates together yield the same bound as in the case $\beta<1$. This completes the proof of Propostion 4.4 .

\section{Appendix A. Besov spaCes and Related faCts}

This appendix provides the definitions of $\Delta_{j}, S_{j}$ and inhomogeneous Besov spaces. Related useful facts such as the Bernstein inequality are also provided here. Materials presented in this appendix here can be found in several books and papers (see e.g. [4],[16] or [84]).

Let $\mathcal{S}\left(\mathbf{R}^{d}\right)$ and $\mathcal{S}^{\prime}\left(\mathbf{R}^{d}\right)$ denote the Schwartz class and tempered distributions, respectively. The partition of unity states that there exist two nonnegative radial functions $\psi, \phi \in \mathcal{S}$ such that

$$
\begin{aligned}
& \operatorname{supp} \psi \subset B\left(0, \frac{11}{12}\right), \quad \operatorname{supp} \phi \subset A\left(0, \frac{3}{4}, \frac{11}{6}\right), \\
& \psi(\xi)+\sum_{j \geq 0} \phi_{j}(\xi)=1 \quad \text { for } \quad \xi \in \mathbf{R}^{d}, \quad \phi_{j}(\xi)=\phi\left(2^{-j} \xi\right), \\
& \operatorname{supp} \psi \cap \operatorname{supp} \phi_{j}=\emptyset \quad \text { if } j \geq 1, \\
& \operatorname{supp} \phi_{j} \cap \operatorname{supp} \phi_{k}=\emptyset \quad \text { if }|j-k| \geq 2,
\end{aligned}
$$

where $B(0, r)$ denotes the ball centered at the origin with radius $r$ and $A\left(0, r_{1}, r_{2}\right)$ the annulus centered at the origin with the inner radius $r_{1}$ and the outer radius $r_{2}$.

For any $f \in \mathcal{S}^{\prime}$, set

$$
\begin{aligned}
& \Delta_{-1} f=\mathcal{F}^{-1}(\psi(\xi) \mathcal{F}(f))=\Psi * f, \\
& \Delta_{j} f=\mathcal{F}^{-1}\left(\phi_{j}(\xi) \mathcal{F}(f)\right)=\Phi_{j} * f, \quad j=0,1,2, \cdots, \\
& \Delta_{j} f=0 \quad \text { for } \quad j \leq-2, \\
& S_{j}=\sum_{k=-1}^{j-1} \Delta_{k} \text { when } j \geq 0,
\end{aligned}
$$

where we have used $\mathcal{F}$ and $\mathcal{F}^{-1}$ to denote the Fourier and inverse Fourier transforms. respectively. Clearly,

$$
\Psi=\mathcal{F}^{-1}(\psi), \quad \Phi_{0}=\Phi=\mathcal{F}^{-1}(\phi), \quad \Phi_{j}(x)=\mathcal{F}^{-1}\left(\phi_{j}\right)(x)=2^{j d} \Phi\left(2^{j} x\right) .
$$


In addition, we can write

$$
\mathcal{F}\left(S_{j} f\right)=\psi\left(\frac{\xi}{2^{j}}\right) \mathcal{F}(f)
$$

With these notation at our disposal, we now provide the definition of the inhomogeneous Besov space.

Definition A.1. For $s \in \mathbf{R}$ and $1 \leq p, q \leq \infty$, the inhomogeneous Besov space $B_{p, q}^{s}$ is defined by

$$
B_{p, q}^{s}=\left\{f \in \mathcal{S}^{\prime}:\|f\|_{B_{p, q}^{s}}<\infty\right\}
$$

where

$$
\|f\|_{B_{p, q}^{s}} \equiv \begin{cases}\left(\sum_{j=-1}^{\infty}\left(2^{j s}\left\|\Delta_{j} f\right\|_{L^{p}}\right)^{q}\right)^{1 / q}, & \text { if } q<\infty, \\ \sup _{-1 \leq j<\infty} 2^{j s}\left\|\Delta_{j} f\right\|_{L^{p}}, & \text { if } q=\infty .\end{cases}
$$

The Besov spaces and the standard Sobolev spaces defined by

$$
W_{p}^{s}=(1-\Delta)^{-s / 2} L^{p}
$$

obey the simple facts stated in the following lemma (see [4]).

Proposition A.2. Assume that $s \in \mathbf{R}$ and $p, q \in[1, \infty]$.

1) If $s_{1} \leq s_{2}$, then $B_{p, q}^{s_{2}} \subset B_{p, q}^{s_{1}}$,

2) If $1 \leq q_{1} \leq q_{2} \leq \infty$, then $B_{p, q_{1}}^{s} \subset B_{p, q_{2}}^{s}$,

3) If $1 \leq p_{1} \leq p_{2} \leq \infty, 1 \leq q_{1}, q_{2} \leq \infty$, and $s_{1} \geq s_{2}+d\left(\frac{1}{p_{1}}-\frac{1}{p_{2}}\right)$, then

$$
B_{p_{1}, q_{1}}^{s_{1}}\left(\mathbf{R}^{d}\right) \subset B_{p_{2}, q_{2}}^{s_{2}}\left(\mathbf{R}^{d}\right)
$$

4) If $1<p<\infty$, then

$$
B_{p, \min (p, 2)}^{s} \subset W_{p}^{s} \subset B_{p, \max (p, 2)}^{s} .
$$

The following Bernstein type inequalities are very useful and have been used in the previous sections.

Proposition A.3. Let $\alpha \geq 0$. Let $1 \leq p \leq q \leq \infty$.

1) If $f$ satisfies

$$
\text { supp } \widehat{f} \subset\left\{\xi \in \mathbf{R}^{d}:|\xi| \leq K 2^{j}\right\},
$$

for some integer $j$ and a constant $K>0$, then

$$
\begin{gathered}
\max _{|\beta|=k}\left\|D^{\beta} f\right\|_{L^{q}\left(\mathbf{R}^{d}\right)} \leq C 2^{k j+j d\left(\frac{1}{p}-\frac{1}{q}\right)}\|f\|_{L^{p}\left(\mathbf{R}^{d}\right)}, \\
\left\|(-\Delta)^{\alpha} f\right\|_{L^{q}\left(\mathbf{R}^{d}\right)} \leq C 2^{2 \alpha j+j d\left(\frac{1}{p}-\frac{1}{q}\right)}\|f\|_{L^{p}\left(\mathbf{R}^{d}\right)}
\end{gathered}
$$

for some constant $C$ depending on $K, p$ and $q$ only. 
2) If $f$ satisfies

$$
\begin{gathered}
\qquad \operatorname{supp} \widehat{f} \subset\left\{\xi \in \mathbf{R}^{d}: K_{1} 2^{j} \leq|\xi| \leq K_{2} 2^{j}\right\} \\
\text { for some integer } j \text { and constants } 0<K_{1} \leq K_{2} \text {, then } \\
\qquad 2^{k j}\|f\|_{L^{q}\left(\mathbf{R}^{d}\right)} \leq \max _{|\beta|=k}\left\|D^{\beta} f\right\|_{L^{q}\left(\mathbf{R}^{d}\right)} \leq C 2^{k j+j d\left(\frac{1}{p}-\frac{1}{q}\right)}\|f\|_{L^{p}\left(\mathbf{R}^{d}\right)}, \\
C 2^{2 \alpha j}\|f\|_{L^{q}\left(\mathbf{R}^{d}\right)} \leq\left\|(-\Delta)^{\alpha} f\right\|_{L^{q}\left(\mathbf{R}^{d}\right)} \leq C 2^{2 \alpha j+j d\left(\frac{1}{p}-\frac{1}{q}\right)}\|f\|_{L^{p}\left(\mathbf{R}^{d}\right)}, \\
\text { where the constants } C \text { depend on } K_{1}, K_{2}, p \text { and } q \text { only. }
\end{gathered}
$$

\section{ACKNOWLEDGEMENTS}

Chae's research was partially supported by NRF grant No.2006-0093854. Constantin's research was partially supported by NSF grant DMS 0804380. Wu's research was partially supported by NSF grant DMS 0907913. Wu thanks the Department of Mathematics at Sungkyunkwan University for its hospitality during his visit there, and thanks Professor Changxing Miao for discussions.

\section{REFERENCES}

[1] H. Abidi and T. Hmidi, On the global well-posedness of the critical quasi-geostrophic equation, SIAM J. Math. Anal. 40 (2008), 167-185.

[2] H. Bae, Global well-posedness of dissipative quasi-geostrophic equations in critical spaces. Proc. Amer. Math. Soc. 136 (2008), 257-261.

[3] B. Barrios, Regularization for the supercritical quasi-geostrophic equation, arXiv:1007.4889v1 [math.AP] 28 Jul 2010.

[4] J. Bergh and J. Löfström, Interpolation Spaces, An Introduction, Springer-Verlag, BerlinHeidelberg-New York, 1976.

[5] W. Blumen, Uniform potential vorticity flow, Part I. Theory of wave interactions and twodimensional turbulence, J. Atmos. Sci. 35 (1978), 774-783.

[6] L. Caffarelli and L. Silvestre, An extension problem related to the fractional Laplacian, Comm. Partial Differential Equations 32 (2007), 1245-1260.

[7] L. Caffarelli and A. Vasseur, Drift diffusion equations with fractional diffusion and the quasigeostrophic equation, Ann. of Math., in press.

[8] J. Carrillo and L. Ferreira, The asymptotic behaviour of subcritical dissipative quasi-geostrophic equations, Nonlinearity 21 (2008), 1001-1018.

[9] D. Chae, The quasi-geostrophic equation in the Triebel-Lizorkin spaces, Nonlinearity 16 (2003), 479-495.

[10] D. Chae, On the continuation principles for the Euler equations and the quasi-geostrophic equation, J. Differential Equations 227 (2006), 640-651.

[11] D. Chae, On the regularity conditions for the dissipative quasi-geostrophic equations, SIAM J. Math. Anal. 37 (2006), 1649-1656.

[12] D. Chae, The geometric approaches to the possible singularities in the inviscid fluid flows, J. Phys. A 41 (2008), 365501, 11 pp.

[13] D. Chae, On the a priori estimates for the Euler, the Navier-Stokes and the quasi-geostrophic equations, Adv. Math. 221 (2009), 1678-1702.

[14] D. Chae, A. Córdoba, D. Córdoba and M. Fontelos, Finite time singularities in a 1D model of the quasi-geostrophic equation, Adv. Math. 194 (2005), 203-223. 
[15] D. Chae and J. Lee, Global well-posedness in the super-critical dissipative quasi-geostrophic equations, Commun. Math. Phys. 233 (2003), 297-311.

[16] J.-Y. Chemin, Perfect Incompressible Fluids, Oxford science publications, Oxford University Press, 1998.

[17] Q. Chen, C. Miao and Z. Zhang, A new Bernstein's inequality and the 2D dissipative quasigeostrophic equation, Commun. Math. Phys. 271 (2007), 821-838.

[18] Q. Chen and Z. Zhang, Global well-posedness of the 2D critical dissipative quasi-geostrophic equation in the Triebel-Lizorkin spaces, Nonlinear Anal. 67 (2007), 1715-1725.

[19] P. Constantin, Euler equations, Navier-Stokes equations and turbulence. Mathematical foundation of turbulent viscous flows, 1-43, Lecture Notes in Math., 1871, Springer, Berlin, 2006.

[20] P. Constantin, D. Córdoba and J. Wu, On the critical dissipative quasi-geostrophic equation, Indiana Univ. Math. J. 50 (2001), 97-107.

[21] P. Constantin, G. Iyer and J. Wu, Global regularity for a modified critical dissipative quasigeostrophic equation, Indiana Univ. Math. J. 57 (2008), 2681-2692.

[22] P. Constantin, M.-C. Lai, R. Sharma, Y.-H. Tseng and J. Wu, New numerical results for the surface quasi-geostrophic equation, preprint.

[23] P. Constantin, A. Majda, and E. Tabak, Formation of strong fronts in the 2-D quasi-geostrophic thermal active scalar, Nonlinearity 7 (1994), 1495-1533.

[24] P. Constantin, Q. Nie and N. Schorghofer, Nonsingular surface quasi-geostrophic flow, Phys. Lett. A 241 (1998), 168-172.

[25] P. Constantin and J. Wu, Behavior of solutions of 2D quasi-geostrophic equations, SIAM J. Math. Anal. 30 (1999), 937-948.

[26] P. Constantin and J. Wu, Regularity of Hölder continuous solutions of the supercritical quasigeostrophic equation, Ann. Inst. H. Poincaré Anal. Non Linéaire 25 (2008), 1103-1110.

[27] P. Constantin and J. Wu, Hölder continuity of solutions of supercritical dissipative hydrodynamic transport equation, Ann. Inst. H. Poincaré Anal. Non Linéaire 26 (2009), 159-180.

[28] D. Córdoba, Nonexistence of simple hyperbolic blow-up for the quasi-geostrophic equation, Ann. of Math. 148 (1998), 1135-1152.

[29] A. Córdoba and D. Córdoba, A maximum principle applied to quasi-geostrophic equations, Commun. Math. Phys. 249 (2004), 511-528.

[30] D. Córdoba and Ch. Fefferman, Behavior of several two-dimensional fluid equations in singular scenarios, Proc. Natl. Acad. Sci. USA 98 (2001), 4311-4312.

[31] D. Córdoba and Ch. Fefferman, Scalars convected by a two-dimensional incompressible flow, Comm. Pure Appl. Math. 55 (2002), 255-260.

[32] D. Córdoba and Ch. Fefferman, Growth of solutions for QG and 2D Euler equations, J. Amer. Math. Soc. 15 (2002), 665-670.

[33] D. Córdoba, M. Fontelos, A. Mancho and J. Rodrigo, Evidence of singularities for a family of contour dynamics equations, Proc. Natl. Acad. Sci. USA 102 (2005), 5949-5952.

[34] M. Dabkowski, Eventual regularity of the solutions to the supercritical dissipative quasi-geostrophic equation, arXiv:1007.2970v1 [math.AP] 18 Jul 2010.

[35] J. Deng, T. Y. Hou, R. Li and X. Yu, Level set dynamics and the non-blowup of the 2D quasigeostrophic equation, Methods Appl. Anal. 13 (2006), 157-180.

[36] B. Dong and Z. Chen, Asymptotic stability of the critical and super-critical dissipative quasigeostrophic equation, Nonlinearity 19 (2006), 2919-2928.

[37] H. Dong, Dissipative quasi-geostrophic equations in critical Sobolev spaces: smoothing effect and global well-posedness, Discrete Contin. Dyn. Syst. 26 (2010), 1197-1211.

[38] H. Dong and D. Du, Global well-posedness and a decay estimate for the critical dissipative quasigeostrophic equation in the whole space, Discrete Contin. Dyn. Syst. 21 (2008), 1095-1101.

[39] H. Dong and D. Li, Finite time singularities for a class of generalized surface quasi-geostrophic equations, Proc. Amer. Math. Soc. 136(2008), 2555-2563.

[40] H. Dong and D. Li, Spatial analyticity of the solutions to the subcritical dissipative quasigeostrophic equations, Arch. Ration. Mech. Anal. 189 (2008), 131-158.

[41] H. Dong and N. Pavlovic, A regularity criterion for the dissipation quasi-geostrophic equation, Ann. Inst. H. Poincaré Anal. Non Linéaire 26 (2009), 1607-1619. 
[42] H. Dong and N. Pavlovic, Regularity criteria for the dissipative quasi-geostrophic equations in H?lder spaces, Comm. Math. Phys. 290 (2009), 801-812.

[43] S. Friedlander, N. Pavlovic and V. Vicol, Nonlinear instability for the critically dissipative quasigeostrophic equation, Comm. Math. Phys. 292 (2009), 797-810.

[44] S. Friedlander and V. Vicol, Global well-posedness for an advection-diffusion equation arising in magneto-geostrophic dynamics, arXiv:1007.1211v1 [math.AP] 12 Jul 2010.

[45] A.E. Gill, Atmosphere-Ocean Dynamics, Academic Press, New York, 1982.

[46] I. Held, R. Pierrehumbert, S. Garner, and K. Swanson, Surface quasi-geostrophic dynamics, J. Fluid Mech. 282 (1995), 1-20.

[47] T. Hmidi and S. Keraani, Global solutions of the super-critical 2D quasi-geostrophic equation in Besov spaces, Adv. Math. 214 (2007), 618-638.

[48] T. Hmidi and S. Keraani, On the global well-posedness of the critical quasi-geostrophic equation, SIAM J. Math. Anal. 40 (2008), 167-185.

[49] N. Ju, The maximum principle and the global attractor for the dissipative 2D quasi-geostrophic equations, Commun. Math. Phys. 255 (2005), 161-181.

[50] N. Ju, Geometric constrains for global regularity of 2D quasi-geostrophic flows, J. Differential Equations 226 (2006), 54-79.

[51] B. Khouider and E. Titi, An inviscid regularization for the surface quasi-geostrophic equation, Comm. Pure Appl. Math. 61 (2008), 1331-1346.

[52] A. Kiselev, Some recent results on the critical surface quasi-geostrophic equation: a review, Hyperbolic problems: theory, numerics and applications, 105-122, Proc. Sympos. Appl. Math., 67, Part 1, AMS, Providence, RI, 2009.

[53] A. Kiselev, Regularity and blow up for active scalars, Math. Model. Math. Phenom. 5 (2010), $225-255$.

[54] A. Kiselev and F. Nazarov, Global regularity for the critical dispersive dissipative surface quasigeostrophic equation, Nonlinearity 23 (2010), 549-554.

[55] A. Kiselev and F. Nazarov, A variation on a theme of Caffarelli and Vasseur, Zap. Nauchn. Sem. POMI 370 (2010), 58-72.

[56] A. Kiselev, F. Nazarov and A. Volberg, Global well-posedness for the critical 2D dissipative quasigeostrophic equation, Invent. Math. 167 (2007), 445-453.

[57] D. Li, Existence theorems for the 2D quasi-geostrophic equation with plane wave initial conditions, Nonlinearity 22 (2009), 1639-1651.

[58] D. Li and J. Rodrigo, Blow up for the generalized surface quasi-geostrophic equation with supercritical dissipation, Comm. Math. Phys. 286 (2009), 111-124.

[59] A. Majda, Introduction to PDEs and Waves for the Atmosphere and Ocean, Courant Lecture Notes 9, Courant Institute of Mathematical Sciences and American Mathematical Society, 2003.

[60] A. Majda and A. Bertozzi, Vorticity and Incompressible Flow, Cambridge University Press, 2002.

[61] A. Majda and E. Tabak, A two-dimensional model for quasigeostrophic flow: comparison with the two-dimensional Euler flow, Phys. D 98 (1996), 515-522.

[62] F. Marchand, Propagation of Sobolev regularity for the critical dissipative quasi-geostrophic equation, Asymptot. Anal. 49 (2006), 275-293.

[63] F. Marchand, Existence and regularity of weak solutions to the quasi-geostrophic equations in the spaces $L^{p}$ or $\dot{H}^{-1 / 2}$, Comm. Math. Phys. 277 (2008), 45-67.

[64] F. Marchand, Weak-strong uniqueness criteria for the critical quasi-geostrophic equation, Phys. D 237 (2008), 1346-1351.

[65] F. Marchand and P.G. Lemarié-Rieusset, Solutions auto-similaires non radiales pour l'équation quasi-géostrophique dissipative critique, C. R. Math. Acad. Sci. Paris 341 (2005), 535-538.

[66] C. Marchirio and M. Pulvirenti, Mathematical Theory of Incompressible Non-viscous Fluids, Springer-Verlag, 1994.

[67] R. May, Global well-posedness for a modified 2D dissipative quasi-geostrophic equation with initial data in the critical Sobolev space $H^{1}$, arXiv:0910.0998v1 [math.AP] 6 Oct 2009.

[68] R. May and E. Zahrouni, Global existence of solutions for subcritical quasi-geostrophic equations, Commun. Pure Appl. Anal. 7 (2008), 1179-1191. 
[69] C. Miao and L. Xue, Global wellposedness for a modified critical dissipative quasi-geostrophic equation, arXiv:0901.1368v4 [math.AP] 18 Sep 2010.

[70] H. Miura, Dissipative quasi-geostrophic equation for large initial data in the critical sobolev space, Commun. Math. Phys. 267 (2006), 141-157.

[71] C. Niche and M. Schonbek, Decay of weak solutions to the 2D dissipative quasi-geostrophic equation, Comm. Math. Phys. 276 (2007), 93-115.

[72] K. Ohkitani and M. Yamada, Inviscid and inviscid-limit behavior of a surface quasigeostrophic flow, Phys. Fluids 9 (1997), 876-882.

[73] J. Pedlosky, Geophysical Fluid Dynamics, Springer, New York, 1987.

[74] J. Reinaud and D. Dritschel, Destructive interactions between two counter-rotating quasigeostrophic vortices, J. Fluid Mech. 639 (2009), 195-211.

[75] S. Resnick, Dynamical problems in nonlinear advective partial differential equations, Ph.D. thesis, University of Chicago, 1995.

[76] J. Rodrigo, The vortex patch problem for the surface quasi-geostrophic equation, Proc. Natl. Acad. Sci. USA 101 (2004), 2684-2686

[77] J. Rodrigo, On the evolution of sharp fronts for the quasi-geostrophic equation, Comm. Pure Appl. Math. 58 (2005), 821-866.

[78] M. Schonbek and T. Schonbek, Asymptotic behavior to dissipative quasi-geostrophic flows, SIAM J. Math. Anal. 35 (2003), 357-375.

[79] M. Schonbek and T. Schonbek, Moments and lower bounds in the far-field of solutions to quasigeostrophic flows, Discrete Contin. Dyn. Syst. 13 (2005), 1277-1304.

[80] L. Silvestre, Eventual regularization for the slightly supercritical quasi-geostrophic equation, Ann. Inst. H. Poincaré Anal. Non Linéaire 27 (2010), no. 2, 693-704.

[81] L. Silvestre, Hölder estimates for advection fractional-diffusion equations, arXiv:1009.5723v1 [math.AP] 29 Sep 2010.

[82] A. Stefanov, Global well-posedness for the 2D quasi-geostrophic equation in a critical Besov space, Electron. J. Differential Equations 2007 (2007), 9 pp.

[83] E. Stein, Singular Integrals and Differentiability Properties of Functions, Princeton Unviersity Press, Princeton, NJ, 1970.

[84] H. Triebel, Theory of Function Spaces, Monographs in Mathematics 78, Birkh?user Verlag, Basel, 1983. $284 \mathrm{pp}$.

[85] H. Wang and H. Jia, Local well-posedness for the 2D non-dissipative quasi-geostrophic equation in Besov spaces, Nonlinear Anal. 70 (2009), 3791-3798.

[86] J. Wu, Quasi-geostrophic-type equations with initial data in Morrey spaces, Nonlinearity 10 (1997), $1409-1420$.

[87] J. Wu, Inviscid limits and regularity estimates for the solutions of the 2-D dissipative quasigeostrophic equations, Indiana Univ. Math. J. 46 (1997), 1113-1124.

[88] J. Wu, Dissipative quasi-geostrophic equations with $L^{p}$ data, Electron. J. Differential Equations 2001 (2001), 1-13.

[89] J. Wu, The quasi-geostrophic equation and its two regularizations, Comm. Partial Differential Equations 27 (2002), 1161-1181.

[90] J. Wu, Global solutions of the 2D dissipative quasi-geostrophic equation in Besov spaces, SIAM J. Math. Anal. 36 (2004/2005), 1014-1030.

[91] J. Wu, The quasi-geostrophic equation with critical or supercritical dissipation, Nonlinearity 18 (2005), 139-154.

[92] J. Wu, Solutions of the 2-D quasi-geostrophic equation in Hölder spaces, Nonlinear Analysis 62 (2005), 579-594.

[93] J. Wu, Lower bounds for an integral involving fractional Laplacians and the generalized NavierStokes equations in Besov spaces, Comm. Math. Phys. 263 (2006), 803-831.

[94] $\mathrm{J} . \mathrm{Wu}$, Existence and uniqueness results for the 2-D dissipative quasi-geostrophic equation, Nonlinear Anal. 67 (2007), 3013-3036.

[95] X. Yu, Remarks on the global regularity for the super-critical 2D dissipative quasi-geostrophic equation, J. Math. Anal. Appl. 339 (2008), 359-371. 
[96] B. Yuan, The dissipative quasi-geostrophic equation in weak Morrey spaces, Acta Math. Sin. (Engl. Ser.) 24 (2008), 253-266.

[97] J. Yuan, On regularity criterion for the dissipative quasi-geostrophic equations, J. Math. Anal. Appl. 340 (2008), 334-339.

[98] Z. Zhang, Well-posedness for the 2D dissipative quasi-geostrophic equations in the Besov space, Sci. China Ser. A 48 (2005), 1646-1655.

[99] Z. Zhang, Global well-posedness for the 2D critical dissipative quasi-geostrophic equation, Sci. China Ser. A 50 (2007), 485-494.

[100] Y. Zhou, Decay rate of higher order derivatives for solutions to the 2-D dissipative quasigeostrophic flows, Discrete Contin. Dyn. Syst. 14 (2006), 525-532.

[101] Y. Zhou, Asymptotic behaviour of the solutions to the 2D dissipative quasi-geostrophic flows, Nonlinearity 21 (2008), 2061-2071.

${ }^{1}$ Department of Mathematics, Sungkyunkwan University, Suwon 440-746, Korea

${ }^{2}$ Department of Mathematics, University of Chicago, 5734 S. University Avenue, Chicago, IL 60637, USA.

${ }^{3}$ Department of Mathematics, Oklahoma State University, 401 Mathematical SciENCES, Stillwater, OK 74078, USA.

E-mail address: chae@skku.edu

E-mail address: const@cs.uchicago.edu

E-mail address: jiahong@math.okstate.edu 